CREAT. MATH. INFORM.

Volume 25 (2016), No. 2,

Pages 141 - 150
Online version at https : //creative-mathematics. cunbm. utcluj.ro/ Print Edition: ISSN 1584 - 286X; Online Edition: ISSN 1843 - 441X

DOI: https://doi.org/10.37193/CMI.2016.02.04

\title{
On the generalized Boolean sum Schurer-Stancu approximation formula
}

\author{
DAN BĂRBOSU and OVIDIU T. POP
}

\begin{abstract}
In this paper, the Schurer-Stancu generalized Boolean sum (GBS, for short) approximation formula is considered and it's remainder term is expressed in terms of bivariate divided differences. When the approximated function is sufficiently smooth, an upper bound estimation for the remainder term is also established. As particular cases, GBS Schurer and respectively GBS Bernstein approximation formulas are obtained and the expressions of their remainder are explicitly given.
\end{abstract}

\section{INTRODUCTION}

We denote $N=\{1,2, \ldots\}$ and $\mathbb{N}_{0}=\mathbb{N} \cup\{0\}$. Let $p \in \mathbb{N}_{0}$ and let $\alpha, \beta$ be real parameters so that $0 \leq \alpha \leq \beta$. The Schurer-Stancu operator [3] is defined for any $m \in \mathbb{N}$, any function $f \in C([0,1+p])$ and any $x \in[0,1+p]$ by:

$$
\left(\widetilde{S}_{m, p}^{(\alpha, \beta)} f\right)(x)=\sum_{k=0}^{m+p} \widetilde{p}_{m k}(x) f\left(\frac{k+\alpha}{m+\beta}\right),
$$

where

$$
\widetilde{p}_{m, k}(x)=\left(\begin{array}{c}
m+p \\
k
\end{array}\right) x^{k}(1-x)^{m-k}
$$

are the fundamental Schurer polynomials [17].

Note that the multiparameter operator (1.1) includes as particular cases other well-known operators: for $\alpha=\beta=0$, the operator (1.1) reduces to the Schurer operator [17]; for $p=0$, (1.1) becomes the Stancu operator [20], while, for $\alpha=\beta=0$ and $p=0,(1.1)$ is the classical Bernstein operator [10].

The method of "parametric extensions" is a very efficient one for constructing multivariate operators [12]. By applying the above mentioned method, in [4] the author considered the following parametric extensions of the operator (1.1):

$$
\begin{aligned}
\left({ }_{x} \widetilde{S}_{m, p}^{(\alpha, \beta)} f\right)(x, y) & =\sum_{k=0}^{m+p} \sum_{j=0}^{n+q} \widetilde{p}_{m, k}(x) \widetilde{p}_{n, j}(y) f\left(\frac{k+\alpha}{m+\beta}, y\right), \\
\left({ }_{y} \widetilde{S}_{n, q}^{(\gamma, \delta)} f\right)(x, y) & =\sum_{k=0}^{m+p} \sum_{j=0}^{n+q} \widetilde{p}_{m, k}(x) \widetilde{p}_{n, j}(y) f\left(x, \frac{j+\gamma}{n+\delta}\right) .
\end{aligned}
$$

Note that in (1.3) and (1.4), $p, q \in \mathbb{N}_{0}, \alpha, \beta, \gamma, \delta$ are real parameters such that $0 \leq \alpha \leq \beta$, $0 \leq \gamma \leq \delta$ and $f:[0,1+p] \times[0,1+q] \rightarrow \mathbb{R}$ is a given real valued function.

Received: 24.04.2016. In revised form: 09.05.2016. Accepted: 26.05.2016

2010 Mathematics Subject Classification. 41A36, 41A80.

Key words and phrases. Schurer-Stancu operator, parametric extension, GBS Schurer-Stancu operator, GBS Schurer operator, GBS Stancu operator, GBS Bernstein operator, GBS Schurer-Stancu approximation formula, divided difference, bivariate divided difference, remainder term.

Corresponding author: Ovidiu T. Pop; ovidiutiberiu@yahoo.com 
The notion of "Generalized Boolean Sum" (GBS, for short) operator was introduced by C. Badea and C. Cottin [1] as the boolean sum of the parametric extensions of a univariate operator.

By using the above definition, the GBS Schurer-Stancu operator is defined for any $m, n \in \mathbb{N}$, any $p, q \in \mathbb{N}_{0}$ and any real parameters $\alpha, \beta, \gamma, \delta$ satisfying $0 \leq \alpha \leq \beta, 0 \leq \gamma \leq \delta$ by:

$$
\begin{aligned}
& \left(\widetilde{U}_{m, p, n, q}^{(\alpha, \beta, \gamma)} f\right)(x, y) \\
& =\sum_{k=0}^{m+p} \sum_{j=0}^{n+q} \widetilde{p}_{m, k}(x) \widetilde{p}_{n, j}(y)\left\{f\left(\frac{k+\alpha}{m+\beta}, y\right)+f\left(x, \frac{j+\gamma}{n+\delta}\right)-f\left(\frac{k+\alpha}{m+\beta}, \frac{j+\gamma}{n+\delta}\right)\right\},
\end{aligned}
$$

for any bounded function $f:[0,1+p] \times[0,1+q] \rightarrow \mathbb{R}$.

Approximation properties of the operator (1.5) were established in [4], [7].

As particular cases of (1.5) we mention the GBS operators of Schurer, Stancu, respectively Bernstein type.

By considering the GBS Schurer-Stancu approximation formula

$$
f=\widetilde{U}_{m, p, n, q}^{(\alpha, \beta, \gamma, \delta)} f+\widetilde{R}_{m, p, n, q}^{(\alpha, \beta, \gamma, \delta)} f,
$$

the first focus of the paper is to express the remainder term $\widetilde{R}_{m, p, n, q}^{(\alpha, \beta, \gamma)} f$ by using the bivariate divided difference of the approximated function.

We start by recalling some results concerning the divided differences. Suppose that $I \subset \mathbb{R}$ is an interval of the real axis, $f: I \rightarrow \mathbb{R}$ is a given function and $x_{0}, x_{1} \in I\left(x_{0} \neq x_{1}\right)$ are given. Then, the divided difference (of first order) of $f$ with respect the distinct knots $x_{0}, x_{1}$ is defined by:

$$
\left[x_{0}, x_{1} ; f\right]=\frac{f\left(x_{1}\right)-f\left(x_{0}\right)}{x_{1}-x_{0}} .
$$

If the knots $x_{0}, x_{1}, \ldots, x_{p} \in I$ are distinct, the $p$-th order divided difference of $f$ with respect to the mentioned distinct knots is defined by the following recurrence formula:

$$
\left[x_{0}, x_{1}, \ldots, x_{p} ; f\right]=\frac{\left[x_{1}, \ldots, x_{p} ; f\right]-\left[x_{0}, \ldots, x_{p-1} ; f\right]}{x_{p}-x_{0}}, p \geq 2 .
$$

For the bivariate divided differences, several definitions were given in [13], [14], [15]. In [8], by using the method of parametric extensions we re-found the definition of the bivariate divided difference.

In the following let $I, J \subset \mathbb{R}$ be intervals, $f: I \times J \rightarrow \mathbb{R}$ be a given function, $p, q \in \mathbb{N}_{0}$, $x_{0}, x_{1}, \ldots, x_{p} \in I$ and $y_{0}, y_{1}, \ldots, y_{q} \in J$ be distinct knots.

If $p, q \in \mathbb{N}, p \geq 2$ and $q \geq 2$, the following recurrence formula

$$
\begin{aligned}
& {\left[\begin{array}{l}
x_{0}, x_{1}, \ldots, x_{p} \\
y_{0}, y_{1}, \ldots, y_{q}
\end{array} ; f\right]=\frac{1}{\left(x_{p}-x_{0}\right)\left(y_{q}-y_{0}\right)}\left(\left[\begin{array}{l}
x_{1}, x_{2}, \ldots, x_{p} \\
y_{1}, y_{2}, \ldots, y_{q}
\end{array} ; f\right]\right.} \\
& \left.\left.-\left[\begin{array}{l}
x_{0}, x_{1}, \ldots, x_{p-1} \\
y_{1}, y_{2}, \ldots, y_{q}
\end{array} ; f\right]-\left[\begin{array}{l}
x_{1}, x_{2}, \ldots, x_{p} \\
y_{0}, y_{1}, \ldots, y_{q-1}
\end{array} ; f\right]+\left[\begin{array}{l}
x_{0}, x_{1}, \ldots, x_{p-1} \\
y_{0}, y_{1}, \ldots, y_{q-1}
\end{array}\right] f\right]\right)
\end{aligned}
$$

holds (see [3]) and

$$
\left[\begin{array}{l}
x_{0}, x_{1}, \ldots, x_{p} \\
y_{0}, y_{1}, \ldots, y_{q}
\end{array} ; f\right]=\left[\begin{array}{l}
x_{i_{0}}, x_{i_{1}}, \ldots, x_{i_{p}} \\
y_{j_{0}}, y_{j_{1}}, \ldots, y_{j_{q}}
\end{array}\right]
$$

where $\left(i_{0}, i_{1}, \ldots, i_{p}\right),\left(j_{0}, j_{1}, \ldots, j_{q}\right)$ are permutations of $(0,1, \ldots, p)$, respectively $(0,1, \ldots, q)$. 
Theorem 1.1. If $q \geq 2$, then

$$
\begin{aligned}
& {\left[\begin{array}{l}
x_{0}, x_{1}, \ldots, x_{p} \\
y_{0}, y_{1}, \ldots, y_{q}
\end{array} ; f\right]} \\
& =\frac{1}{y_{q}-y_{q-1}}\left(\left[\begin{array}{l}
x_{0}, x_{1}, \ldots, x_{p} \\
y_{0}, y_{1}, \ldots, y_{q-2}, y_{q}
\end{array} ; f\right]-\left[\begin{array}{l}
x_{0}, x_{1}, \ldots, x_{p} \\
y_{0}, y_{1}, \ldots, y_{q-1}
\end{array} ; f\right]\right)
\end{aligned}
$$

and, if $p \geq 2$, then

$$
\begin{aligned}
& {\left[\begin{array}{l}
x_{0}, x_{1}, \ldots, x_{p} \\
y_{0}, y_{1}, \ldots, y_{q}
\end{array} ; f\right]} \\
& =\frac{1}{x_{p}-x_{p-1}}\left(\left[\begin{array}{l}
x_{0}, x_{1}, \ldots, x_{p-2}, x_{p} \\
y_{0}, y_{1}, \ldots, y_{q}
\end{array} ; f\right]-\left[\begin{array}{l}
x_{0}, x_{1}, \ldots, x_{p-1} \\
y_{0}, y_{1}, \ldots, y_{q}
\end{array} ; f\right]\right) .
\end{aligned}
$$

Proof. By using the method of parametric extensions (see [8]) and (1.10), we have that

$$
\begin{gathered}
{\left[\begin{array}{l}
x_{0}, x_{1}, \ldots, x_{p} \\
y_{0}, y_{1}, \ldots, y_{q}
\end{array} ; f\right]=\left[\begin{array}{l}
x_{0}, x_{1}, \ldots, x_{p} \\
y_{q-1}, y_{0}, y_{1}, \ldots, y_{q-2}, y_{q}
\end{array} ; f\right]} \\
=\left[x_{0} ;\left[y_{q-1}, y_{0}, y_{1}, \ldots, y_{q-2}, y_{q} ; f\right]_{y}\right]_{x} \\
\left.y_{0}, x_{1}, \ldots, x_{p} \frac{\left[y_{0}, y_{1}, \ldots, y_{q-2}, y_{q} ; f\right]_{y}-\left[y_{q-1}, y_{0}, y_{1}, \ldots, y_{q-2} ; f\right]_{y}}{y_{q}}\right]_{x} \\
=\frac{1}{y_{q}-y_{q-1}}\left(\left[x_{0}, x_{1}, \ldots, x_{p} ;\left[y_{0}, y_{1}, \ldots, y_{q-2}, y_{q} ; f\right]_{y}\right]_{x}\right. \\
\left.-\left[x_{0}, x_{1}, \ldots, x_{p} ;\left[y_{q-1}, y_{0}, y_{1}, \ldots, y_{q-2} ; f\right]_{y}\right]_{x}\right) \\
=\frac{1}{y_{q}-y_{q-1}}\left(\left[\begin{array}{l}
x_{0}, x_{1}, \ldots, x_{p} \\
y_{0}, y_{1}, \ldots, y_{q-2}, y_{q}
\end{array} ; f\right]-\left[\begin{array}{l}
x_{0}, x_{1}, x_{2}, \ldots, x_{p} \\
y_{q-1}, y_{0}, y_{1}, \ldots, y_{q-2}
\end{array} ; f\right]\right),
\end{gathered}
$$

which yields the identity (1.11).

In a similar way, one proves (1.12).

From Theorem 1.1, the following identities

$$
\begin{aligned}
& {\left[\begin{array}{l}
x_{0}, x_{1}, x_{2} \\
y_{0}, y_{1}
\end{array} ; f=\frac{1}{x_{2}-x_{1}}\left(\left[\begin{array}{l}
x_{0}, x_{2} \\
y_{0}, y_{1}
\end{array} ; f\right]-\left[\begin{array}{l}
x_{0}, x_{1} \\
y_{0}, y_{1}
\end{array} ; f\right]\right),\right.} \\
& {\left[\begin{array}{l}
x_{0}, x_{1} \\
y_{0}, y_{1}, y_{2}
\end{array} ; f\right]=\frac{1}{y_{2}-y_{1}}\left(\left[\begin{array}{l}
x_{0}, x_{1} \\
y_{0}, y_{2}
\end{array} ; f\right]-\left[\begin{array}{l}
x_{0}, x_{1} \\
y_{0}, y_{1}
\end{array} ; f\right]\right),}
\end{aligned}
$$

hold and from (1.9) we have

$$
\begin{aligned}
{\left[\begin{array}{l}
x_{0}, x_{1}, x_{2} \\
y_{0}, y_{1}, y_{2}
\end{array} ; f\right] } & =\frac{1}{\left(x_{2}-x_{1}\right)\left(y_{2}-y_{1}\right)}\left(\left[\begin{array}{l}
x_{0}, x_{2} \\
y_{0}, y_{2}
\end{array} ; f\right]-\left[\begin{array}{l}
x_{0}, x_{1} \\
y_{0}, y_{2}
\end{array} ; f\right]\right. \\
& \left.-\left[\begin{array}{l}
x_{0}, x_{2} \\
y_{0}, y_{1}
\end{array} ; f\right]+\left[\begin{array}{l}
x_{0}, x_{1} \\
y_{0}, y_{1}
\end{array} ; f\right]\right) .
\end{aligned}
$$

We shall use these identities in the proof of Theorem 2.3. Now, we recall the following theorem from [9].

Theorem 1.2. Let $p, q \in \mathbb{N}, a \leq x_{0}<x_{1}<\cdots<x_{p} \leq b, c \leq y_{0}<y_{1}<\cdots<y_{q} \leq d$ and $f:[a, b] \times[c, d] \rightarrow \mathbb{R}$. If $f \in C^{(p-1, q-1)}([a, b] \times[c, d])$ and exists $\frac{\partial^{p+q} f}{\partial x^{p} \partial y^{q}}$ on $] a, b[\times] c, d[$ then, there exists $(\xi, \eta) \in] a, b[\times] c, d[$ such that

$$
\left[\begin{array}{l}
x_{0}, x_{1}, \ldots, x_{p} \\
y_{0}, y_{1}, \ldots, y_{q}
\end{array} ; f\right]=\frac{1}{p ! q !} \frac{\partial^{p+q} f}{\partial x^{p} \partial y^{q}}(\xi, \eta) .
$$




\section{MAIN RESUlts}

Let $m, n \in \mathbb{N}, p, q \in \mathbb{N}_{0}$ and $f:[0,1+p] \times[0,1+q] \rightarrow \mathbb{R}$ be a given function. In what follows, first we shall prove:

Theorem 2.3. The remainder term of the approximation formula (1.6) can be represented under the form:

$$
\begin{gathered}
\left(\widetilde{R}_{m, p, n, q}^{(\alpha, \beta, \gamma, \delta)} f\right)(x, y) \\
=x y(1-x)(1-y) \frac{(m+p)(n+q)}{(m+\beta)^{2}(n+\delta)^{2}} \sum_{k=0}^{m+p-1} \sum_{j=0}^{n+q-1} \widetilde{p}_{m-1, k}(x) \widetilde{p}_{n-1, j}(y) \\
\times\left[\begin{array}{l}
x, \frac{k+\alpha}{m+\beta}, \frac{k+\alpha+1}{m+\beta} \\
y, \frac{j+\gamma}{n+\delta}, \frac{j+\gamma+1}{n+\delta}
\end{array} ; f\right]-\frac{m+p}{(m+\beta)^{2}(n+\delta)} x(1-x)\{(\delta-q) y-\gamma\} \sum_{k=0}^{m+p-1} \sum_{j=0}^{n+q} \\
\times \widetilde{p}_{m-1, k}(x) \widetilde{p}_{n, j}(y)\left[\begin{array}{c}
x, \frac{k+\alpha}{m+\beta}, \frac{k+\alpha+1}{m+\beta} \\
y, \frac{j+\gamma}{n+\delta}
\end{array}\right]-\frac{n+q}{(m+\beta)(n+\delta)^{2}} y(1-y)\{(\beta-p) x-\alpha\} \\
\times \sum_{k=0}^{m+p} \sum_{j=0}^{n+q-1} \widetilde{p}_{m, k}(x) \widetilde{p}_{n-1, j}(y)\left[y, \frac{j+\gamma}{n+\delta}, \frac{j+\gamma+1}{n+\delta} ; f\right]+\frac{1}{(m+\beta)(n+\delta)} \\
\times\{(\beta-p) x-\alpha\}\{(\delta-q) y-\gamma\} \sum_{k=0}^{m+p} \sum_{j=0}^{n+q} \widetilde{p}_{m, k}(x) \widetilde{p}_{n, j}(y)\left[\begin{array}{c}
x, \frac{k+\alpha}{m+\beta} \\
y, \frac{j+\gamma}{n+\delta}
\end{array}\right],
\end{gathered}
$$

where $x \in[0,1+p] \backslash\left\{\frac{k+\alpha}{m+\beta}, k \in\{0,1, \ldots, m+p\}\right\}$ and $y \in[0,1+q] \backslash\left\{\frac{j+\gamma}{n+\delta}, j \in\right.$ $\{0,1, \ldots, n+q\}\}$.

Proof. From (1.6) and (1.5) we get,

$$
\begin{aligned}
& \left(\widetilde{R}_{m, p, n, q}^{(\alpha, \beta, \gamma, \delta)} f\right)(x, y) \\
& =\sum_{k=0}^{m+p} \sum_{j=0}^{n+q} \widetilde{p}_{m, k}(x) \widetilde{p}_{n, j}(y)\left(x-\frac{k+\alpha}{m+\beta}\right)\left(y-\frac{j+\gamma}{n+\delta}\right)\left[\begin{array}{c}
x, \frac{k+\alpha}{m+\beta} \\
y, \frac{j+\gamma}{n+\delta}
\end{array} ; f\right] \\
& =\frac{1}{(m+\beta)(n+\delta)} \sum_{k=0}^{m+p} \sum_{j=0}^{n+q} \widetilde{p}_{m, k}(x) \widetilde{p}_{n, j}(y) \\
& \times\{(m+\beta) x-(k+\alpha)\}\{(n+\delta) y-(j+\gamma)\}\left[\begin{array}{c}
x, \frac{k+\alpha}{m+\beta} \\
y, \frac{j+\gamma}{n+\delta}
\end{array} ; f\right] .
\end{aligned}
$$

Next, by using the following simple identities

$$
\begin{gathered}
(m+\beta) x-(k+\alpha)=(m+p-k) x+(\beta-p) x-\alpha-k(1-x), \\
(n+\delta) y-(j+\gamma)=(n+q-j) y+(\delta-q) y-\gamma-j(1-y),
\end{gathered}
$$


after some elementary computations, by means of the relations (1.13)-(1.15), one obtains

$$
\begin{gathered}
\left(\widetilde{R}_{m, p, n, q}^{(\alpha, \beta, \gamma, \delta)} f\right)(x, y) \\
=x y(1-x)(1-y) \frac{(m+p)(n+q)}{(m+\beta)^{2}(n+\delta)^{2}} \sum_{k=0}^{m+p-1} \sum_{j=0}^{n+q-1} \widetilde{p}_{m-1, k}(x) \widetilde{p}_{n-1, j}(y) \\
\times\left[\begin{array}{l}
x, \frac{k+\alpha}{m+\beta}, \frac{k+\alpha+1}{m+\beta} \\
y, \frac{j+\gamma}{n+\delta}, \frac{j+\delta+1}{n+\delta}
\end{array}\right]-\frac{m+p}{(m+\beta)^{2}(n+\delta)} x(1-x)\{(\delta-q) y-\gamma\} \\
\times \sum_{k=0}^{m+p-1} \sum_{j=0}^{n+q} \widetilde{p}_{m-1, k}(x) \widetilde{p}_{n, j}(y)\left[\begin{array}{l}
x, \frac{k+\alpha}{m+\beta}, \frac{k+\alpha+1}{m+\beta} \\
y, \frac{j+\gamma}{n+\delta}
\end{array}\right]-\frac{n+q}{(m+\beta)(n+\delta)^{2}} \\
\times y(1-y)\{(\beta-p) x-\alpha\} \sum_{k=0}^{m+p} \sum_{j=0}^{n+q-1} \widetilde{p}_{m, k}(x) \widetilde{p}_{n-1, j}(y)\left[\begin{array}{l}
x, \frac{k+\alpha}{m+\beta} \\
y, \frac{j+\gamma}{n+\delta}, \frac{j+\gamma+1}{n+\delta}
\end{array}\right] \\
+\frac{1}{(m+\beta)(n+\delta)}\{(\beta-p) x-\alpha\}\{(\delta-q) y-\gamma\} \\
\times \sum_{k=0}^{m+p} \sum_{j=0}^{n+q} \widetilde{p}_{m, k}(x) \widetilde{p}_{n, j}(y)\left[\begin{array}{l}
x, \frac{k+\alpha}{m+\beta} \\
y, \frac{j+\gamma}{n+\delta}
\end{array} ; f\right]
\end{gathered}
$$

which is the desired equality (2.17).

Remark 2.1. For $\alpha=\beta=\gamma=\delta=0$, the GBS Schurer-Stancu approximation formula (1.6) becomes the following Schurer GBS approximation formula:

$$
f=\widetilde{U}_{m, p, n, q} f+\widetilde{R}_{m, p, n, q} f .
$$

By applying Theorem 2.3, the following corollary follows.

Corollary 2.1. The remainder term of the GBS Schurer approximation formula can be represented under the form:

$$
\begin{gathered}
\left(\widetilde{R}_{m, p, n, q} f\right)(x, y) \\
=x y(1-x)(1-y) \frac{(m+p)(n+q)}{m^{2} n^{2}} \sum_{k=0}^{m+p-1} \sum_{j=0}^{n+q-1} \widetilde{p}_{m-1, k}(x) \widetilde{p}_{n-1, j}(y)\left[\begin{array}{l}
x, \frac{k}{m}, \frac{k+1}{m} \\
y, \frac{j}{n}, \frac{j+1}{n}
\end{array} ; f\right] \\
+\frac{m+p}{m^{2} n} x(1-x) q y \sum_{k=0}^{m+p-1} \sum_{j=0}^{n+q} \widetilde{p}_{m-1, k} \widetilde{p}_{n, j}(y)\left[\begin{array}{c}
x, \frac{k}{m}, \frac{k+1}{m} \\
y, \frac{j}{n}
\end{array} ; f\right] \\
+\frac{n+q}{m n^{2}} y(1-y) p x \sum_{k=0}^{m+p} \sum_{j=0}^{n+q-1} \widetilde{p}_{m, k}(x) \widetilde{p}_{n-1, j}(y)\left[\begin{array}{c}
x, \frac{k}{m} \\
y, \frac{j}{n}, \frac{j+1}{n}
\end{array}\right] f \\
+\frac{p q}{m n} x y \sum_{k=0}^{m+p} \sum_{j=0}^{n+q} \widetilde{p}_{m, k}(x) \widetilde{p}_{n, j}(y)\left[\begin{array}{l}
x, \frac{k}{m} \\
y, \frac{j}{n}
\end{array}\right],
\end{gathered}
$$

where $x \in[0,1+p] \backslash\left\{\frac{k}{m}, k \in\{0,1, \ldots, m+p\}\right\}$ and

$$
y \in[0,1+q] \backslash\left\{\frac{j}{n}, j \in\{0,1, \ldots, n+q\}\right\} .
$$


Remark 2.2. For $p=q=0$, the GBS Schurer-Stancu approximation formula (1.6) reduces to the following Stancu GBS approximation formula:

$$
f=\widetilde{U}_{m, n}^{(\alpha, \beta, \gamma, \delta)} f+\widetilde{R}_{m, n}^{(\alpha, \beta, \gamma, \delta)} f .
$$

From Theorem 2.3, we also obtain

Corollary 2.2. The remainder term of the GBS Stancu approximation formula (2.20) can be represented under the form:

$$
\begin{gathered}
\left(\widetilde{R}_{m, n}^{(\alpha, \beta, \gamma, \delta)} f\right)(x, y) \\
\left.=x y(1-x)(1-y) \frac{m n}{(m+\beta)^{2}(n+\delta)^{2}} \sum_{k=0}^{m-1} \sum_{j=0}^{n-1} p_{m-1, k}(x) p_{n-1, j}(y)\left[\begin{array}{l}
x, \frac{k+\alpha}{m+\beta}, \frac{k+\alpha+1}{m+\beta} \\
y, \frac{j+\gamma}{n+\delta}, \frac{j+\gamma+1}{n+\delta}
\end{array}\right] f\right] \\
\left.-\frac{m}{(m+\beta)^{2}(n+\delta)} x(1-x)(\delta y-\gamma) \sum_{k=0}^{m-1} \sum_{j=0}^{n} p_{m-1, k}(x) p_{n, j}(y)\left[\begin{array}{l}
x, \frac{k+\alpha}{m+\beta}, \frac{k+\alpha+1}{m+\beta} \\
y, \frac{j+\gamma}{n+\delta}
\end{array}\right] f\right] \\
-\frac{n}{(m+\beta)(n+\delta)^{2}} y(1-y)(\beta x-\alpha) \sum_{k=0}^{m} \sum_{j=0}^{n-1} p_{m, k}(x) p_{n-1, j}(y)\left[\begin{array}{l}
x, \frac{k+\alpha}{m+\beta} \\
y, \frac{j+\gamma}{n+\delta}, \frac{j+\gamma+1}{n+\delta}
\end{array}\right] f \\
+\frac{1}{(m+\beta)(n+\delta)}(\beta x-\alpha)(\delta y-\gamma) \sum_{k=0}^{m} \sum_{j=0}^{n} p_{m, k}(x) p_{n, j}(y)\left[\begin{array}{l}
m+\beta \\
y, \frac{j+\gamma}{n+\delta}
\end{array}\right],
\end{gathered}
$$

where:

$$
p_{m, k}(x)=\left(\begin{array}{c}
m \\
k
\end{array}\right) x^{k}(1-x)^{m-k}, p_{n, j}(y)=\left(\begin{array}{c}
n \\
j
\end{array}\right) y^{j}(1-y)^{n-j},
$$

are the fundamental Bernstein polynomials and $x \in[0,1] \backslash\left\{\frac{k+\alpha}{m+\beta}, k \in\{0,1, \ldots, m\}\right\}, y \in[0,1] \backslash\left\{\frac{j+\gamma}{n+\delta}, j \in\{0,1, \ldots, n\}\right\}$.

Remark 2.3. For $p=q=0$ and $\alpha=\beta=\gamma=\delta=0$, the GBS approximation formula (1.6) is the classical GBS Bernstein approximation formula:

$$
f=U_{m, n} f+R_{m, n} f,
$$

first considered by E. Dobrescu and I. Matei [7]. For other related results see [1], [10], [11], [12], [13], [14], [15], [16], [17], [21], [23], [26], [27], [28], [29], [32] and [39].

By applying Theorem 2.3, it follows:

Corollary 2.3. The remainder term of the GBS Bernstein approximation formula (2.22) can be represented under the form:

$$
\begin{aligned}
& \left(R_{m, n} f\right)(x, y) \\
& =\frac{x y(1-x)(1-y)}{m n} \sum_{k=0}^{m-1} \sum_{j=0}^{n-1} p_{m-1, k}(x) p_{n-1, j}(y)\left[\begin{array}{l}
x, \frac{k}{m}, \frac{k+1}{m} \\
y, \frac{j}{n}, \frac{j+1}{n}
\end{array} ; f\right]
\end{aligned}
$$

where $x \in[0,1] \backslash\left\{\frac{k}{m}, k \in\{0,1, \ldots, m\}\right\}$ and $y \in[0,1] \backslash\left\{\frac{j}{n}, j \in\{0,1, \ldots, n\}\right\}$. 
Theorem 2.4. Let $f:[0,1+p] \times[0,1+q] \rightarrow \mathbb{R}$ be a function with the property that $f \in$ $C^{(2,2)}([0,1+p] \times[0,1+q])$. Then, for any $(x, y) \in[0,1] \times[0,1]$ and any $m, n \in \mathbb{N}$, we have

$$
\begin{aligned}
\left|\left(\widetilde{R}_{m, p, n, q}^{\alpha, \beta, \gamma, \delta)} f\right)(x, y)\right| & \leq \frac{x y(1-x)(1-y)}{4} \frac{(m+p)(n+q)}{(m+\beta)^{2}(n+\delta)^{2}} M_{1}(f) \\
& +\frac{x(1-x)|(\delta-q) y-\gamma|}{2} \frac{m+p}{(m+\beta)^{2}(n+\delta)} M_{2}(f) \\
& +\frac{y(1-y)|(\beta-p) x-\alpha|}{2} \frac{n+q}{(m+\beta)(n+\delta)^{2}} M_{3}(f) \\
& +|(\beta-p) x-\alpha||(\delta-q) y-\gamma| \frac{1}{(m+\beta)(n+\delta)} M_{4}(f) \\
& \leq \frac{(m+p)(n+q)}{64 m^{2} n^{2}} M_{1}(f)+\frac{m+p}{8 m^{2} n} m_{2} M_{2}(f) \\
& +\frac{n+q}{8 m n^{2}} m_{1} M_{3}(f)+\frac{1}{m n} m_{1} m_{2} M_{4}(f)
\end{aligned}
$$

and

$$
\left|\left(\widetilde{R}_{m, p, n, q} f\right)(x, y)\right| \leq \frac{(9 m+p)(9 n+q)}{64 m^{2} n^{2}} M(f)
$$

where

$$
\begin{aligned}
& M_{1}(f)=\sup _{(x, y) \in[0,1+p] \times[0,1+q]}\left|\frac{\partial^{4} f}{\partial x^{2} \partial y^{2}}(x, y)\right|, \\
& M_{2}(f)=\sup _{(x, y) \in[0,1+p] \times[0,1+q]}\left|\frac{\partial^{3} f}{\partial x^{2} \partial y}(x, y)\right|, \\
& M_{3}(f)=\sup _{(x, y) \in[0,1+p] \times[0,1+q]}\left|\frac{\partial^{3} f}{\partial x \partial y^{2}}(x, y)\right|, \\
& M_{4}(f)=\sup _{(x, y) \in[0,1+p] \times[0,1+q]}\left|\frac{\partial^{2} f}{\partial x \partial y}(x, y)\right|,
\end{aligned}
$$

$m_{1}=\max \{\alpha,|\beta-p-\alpha|\}, m_{2}=\max \{\gamma,|\delta-q-\gamma|\}$ and

$$
M(f)=\max \left\{M_{1}(f), m_{2} M_{2}(f), m_{1} M_{3}(f), m_{1} m_{2} M_{4}(f)\right\}
$$

where $x \in[0,1+p] \backslash\left\{\frac{k+\alpha}{m+\beta}, k \in\{0,1, \ldots, m+p\}\right\}$ and

$$
y \in[0,1+q] \backslash\left\{\frac{j+\gamma}{n+\delta}, j \in\{0,1, \ldots, n+q\}\right\} .
$$

Proof. In (2.17) we apply Theorem 1.2 and we have that

$\left(\xi_{1}(k, j), \eta_{1}(k, j)\right),\left(\xi_{2}(k, j), \eta_{2}(k, j)\right),\left(\xi_{3}(k, j), \eta_{3}(k, j)\right),\left(\xi_{4}(k, j), \eta_{4}(k, j)\right)$ 
$\in] 0,1+p[\times] 0,1+q[$ exist so that

$$
\begin{aligned}
& \left(\widetilde{R}_{m, p, n, q}^{(\alpha, \beta, \gamma, \delta)} f\right)(x, y)=x y(1-x)(1-y) \frac{(m+p)(n+q)}{(m+\beta)^{2}(n+\delta)^{2}} \\
& \times \sum_{k=0}^{m+p-1} \sum_{j=0}^{n+q-1} \widetilde{p}_{m-1, k}(x) \widetilde{p}_{n-1, j}(y) \frac{1}{2 ! 2 !} \frac{\partial^{4} f}{\partial x^{2} \partial y^{2}}\left(\xi_{1}(k, j), \eta_{1}(k, j)\right) \\
& -\frac{m+p}{(m+\beta)^{2}(n+\delta)} x(1-x)\{(\delta-q) y-\gamma\} \\
& \times \sum_{k=0}^{m+p-1} \sum_{j=0}^{n+q} \widetilde{p}_{m-1, k}(x) \widetilde{p}_{n, j}(y) \frac{1}{2 ! 1 !} \frac{\partial^{3} f}{\partial x^{2} \partial y}\left(\xi_{2}(k, j), \eta_{2}(k, j)\right) \\
& -\frac{n+q}{(m+\beta)(n+\delta)^{2}} y(1-y)\{(\beta-p) x-\alpha\} \\
& \times \sum_{k=0}^{m+p} \sum_{j=0}^{n+q-1} \widetilde{p}_{m, k}(x) \widetilde{p}_{n-1, j}(y) \frac{1}{1 ! 2 !} \frac{\partial^{3} f}{\partial x \partial y^{2}}\left(\xi_{3}(k, j), \eta_{3}(k, j)\right) \\
& +\frac{1}{(m+\beta)(n+\delta)}\{(\beta-p) x-\alpha\}\{(\delta-q) y-\gamma\} \\
& \times \sum_{k=0}^{m+p} \sum_{j=0}^{n+q} \widetilde{p}_{m, k}(x) \widetilde{p}_{n, j}(y) \frac{\partial^{2} f}{\partial x \partial y}\left(\xi_{4}(k, j), \eta_{4}(k, j)\right) .
\end{aligned}
$$

Since $x(1-x) \leq \frac{1}{4}, y(1-y) \leq \frac{1}{4}$, we obtain

$$
\begin{aligned}
& \sum_{k=0}^{m+p} \sum_{j=0}^{n+q} \widetilde{p}_{m, k}(x) \widetilde{p}_{n, j}(y)=\sum_{k=0}^{m+p-1} \sum_{j=0}^{n+q} \widetilde{p}_{m-1, k}(x) \widetilde{p}_{n, j}(y) \\
& =\sum_{k=0}^{m+p} \sum_{j=0}^{n+q-1} \widetilde{p}_{m, k}(x) \widetilde{p}_{n-1, j}(y)=\sum_{k=0}^{m+p-1} \sum_{j=0}^{n+q-1} \widetilde{p}_{m-1, k}(x) \widetilde{p}_{n-1, j}(y)=1,
\end{aligned}
$$

By taking the absolute value in the relation above and by using the fact that the partial derivatives of $f$ are bounded on $[0,1+p] \times[0,1+q],|(\beta-p) x-\alpha| \leq \max \{\alpha,|\beta-p-\alpha|\}$ for any $x \in[0,1]$ and $|(\delta-q) y-\gamma| \leq \max \{\gamma,|\delta-q-\gamma|\}$ for any $y \in[0,1]$, we get the inequalities from (2.24). From (2.24), it follows (2.25).

Remark 2.4. From (1.6) and (2.25) it follows that in the conditions of Theorem 2.2, the sequence $\left(\widetilde{U}_{m, p, n, q} f\right)_{m, n \geq 1}$ converges uniformly to function $f$ on $[0,1] \times[0,1]$.

\section{REFERENCES}

[1] Agrawal, P. N., Gupta, V. and Sathish Kumar, A., On q-analogue of Bernstein-Schurer-Stancu operators, Appl. Math. Comput., 219 (2013), No. 14, 7754-7764

[2] Badea, C. and Cottin, C., Korovkin-type Theorems for Generalized Boolean sum operators, Colloquia Mathematica Societatis Janos Bolyai, 58, Approximation Theory, Kecskemét (Hungary) (1990), 51-67

[3] Bărbosu, D., The approximation of multivariate functions using boolean sums of linear operators of interpolating type (Romanian), Ed. Risoprint, Cluj-Napoca, 2002

[4] Bărbosu, D., Schurer-Stancu type operators, Studia Univ. Babeş-Bolyai Math., 48 (2003), No. 3, 31-35

[5] Bărbosu, D., GBS operators of Schurer-Stancu type, An. Univ. Craiova Ser. Mat. Inform., 31 (2003), 1-7

[6] Bărbosu, D., On the Schurer-Stancu approximation formula, Carpathian J. Math., 21 (2005), No. 1-2, 7-12

[7] Bărbosu, D., Approximation properties of a bivariate Stancu type operator, Rev. Anal. Numér. Théor. Approx., 34 (2005), No. 1, 17-21 
[8] Bărbosu, D., Polynomial approximation by means of Schurer-Stancu type operators, Ed. Univ. de Nord, Baia Mare, 2006

[9] Bărbosu, D., Two dimensional divided differences revisited, Creative Math. Inform., 17 (2008), 1-7

[10] Bărbosu, D., A Schurer-Stancu type quadrature formula, Carpathian J. Math., 23 (2007), No. 1-2, 27-31

[11] Bărbosu, D, Simultaneous approximation by bivariate Schurer-Stancu type operators, Math. Balk., New Ser., 20 (2006) No. 3-4, 351-358

[12] Bărbosu, D., The Voronvskaja theorem for the Schurer-Stancu operators, Bul. Ştiinţ. Univ. Politeh. Timiş., Ser. Mat.-Fiz., 50 (64) (2005), No. 1, 27-31

[13] Bărbosu, D., A survey on the approximation properties of Schurer-Stancu operators, Carpathian J. Math., 20 (2004), No. 1, 1-5

[14] Bărbosu, D., The Kantorovich forms of Schurer-Stancu operators, Demonstr. Math., 37 (2004), No. 2, 383-391

[15] Bărbosu, D., The Schurer-Stancu approximation formula revisited, Creat. Math. Inform., 22 (2013), No. 1, 15-18

[16] Bărbosu, D. and Pop, O. T., Bivariate Schurer-Stancu operators revisited, Carpathian J. Math., 26 (2010), No. 1, 24-35

[17] Bărbosu, D. and Pop, O, T., A cubature formula of Schurer-Stancu type, Creat. Math. Inform., 18 (2009), No. 2, 103-109

[18] Bărbosu, D. and Pop, O. T., A note on the GBS Bernstein's approximation formula, An. Univ. Craiova Ser. Mat. Inform., 35 (2008), 1-6

[19] Bernstein, S. N., Demonstration du théorème de Weierstrass fondé sur le calcul des probabilités, Common. Soc. Math. Kharkhow (2), 13 (1912-1913), 1-2

[20] Delvos, F. J. and Schempp, W., Boolean Methods in Interpolation and Approximation, Longman Scientific \& Technical, New York, 1989

[21] Fărcaş, M. D., About some bivariate operators of Schurer-Stancu type, Int. J. Pure Appl. Math., 50 (2009), No. 4, 505-514

[22] Gordon, W. J., Distributive lattices and the approximation of multivariate functions, in Proc. Symp. Approximation with Emphasis on Spline Functions, ed. by I. J. Schoenberg, Acad. Press, New-York (Madison, Wisc. 1969), 223-277

[23] Indrea, A. D., Indrea, A. and Braica, P. I., Note on a Schurer-Stancu-type operator, Creat. Math. Inform., 24 (2015), No. 1, 61-67

[24] Ionescu, D. V., Divided differences (in Romanian), Ed. Academiei R.S.R., Bucureşti, 1978

[25] Ivan, M., Elements of Interpolation Theory, Mediamira Science Publisher, Cluj-Napoca, 2004

[26] Kang, S. M, Acu, A. M., Rafiq, A. and Kwun, Y. Ch., On q-analogue of Stancu-Schurer-Kantorovich operators based on q-Riemann integral, J. Comput. Anal. Appl., 21 (2016), No. 3, 564-577

[27] Miclăuş, D., The generalization of some results for Schurer and Schurer-Stancu operators, Rev. Anal. Numér. Théor. Approx., 40 (2011), No. 1, 52-63

[28] Muraru, C. V., Note on the q-Stancu-Schurer operator, Miskolc Math. Notes, 14 (2013), No. 1, 191-200

[29] Özarslan, M. A. and Vedi, T., Direct and inverse theorems for multivariate Bernstein-Schurer-Stancu operators, Miskolc Math. Notes, 16 (2016), No. 2, 1073-1089

[30] Popoviciu, T., Sur quelques proprietés des fonctions d'une ou de deux variables réelles, Mathematica (1934), 1-85

[31] Popoviciu, T., Sur le reste dans certain formules lineaires d'approximation de l'analyse, Mathematica I (24) (1952), 95-142

[32] Ren, M. Y. and Zeng, X. M., Approximation by complex Schurer-Stancu operators in compact disks, J. Comput. Anal. Appl., 15 (2013), No. 5, 833-843

[33] Schurer, F., Linear positive operators in approximation theory, Math. Inst. Techn. Univ. Delft: Report, 1962

[34] Stancu, D. D., On the remainder term in approximation formulas by Bernstein polynomials, Notices, Amer. Math. Soc. 9, 20 (1962)

[35] Stancu, D. D., Evaluation of the remainder term in approximation formulas by Bernstein polynomials, Math. Comput., 17 (1963), 270-278.

[36] Stancu, D. D., Approximation of functions by a new class of linear polinomial operators, Rev. Roum. Math. Pures et Appl., 13 (1968), No. 8, 1173-1194

[37] Stancu, D. D., A note on the remainder term in a polynomial approximation formula, Studia Univ. Babeş-Bolyai Math., 41 (1996), 95-101

[38] Stancu, D. D., Coman, Gh. and Blaga, P., Numerical Analysis and Approximation Theory (in Romanian), I, Presa Univ. Clujeană, Cluj-Napoca, 2001

[39] Vedi, T. and Özarslan, M. A., Chlodowsky variant of q-Bernstein-Schurer-Stancu operators, J. Inequal. Appl., 2014, Article ID 189, 14 p., electronic only (2014) 
Department OF MATHEMATICS AND COMPUTER SCIENCE

North University CENTER AT BAIA MARE

TECHNICAL UNIVERSITY OF CLUJ-NAPOCA

VICTORIEI 76, 430122 BAIA MARE ROMANIA

Email address: barbosudan@yahoo.com

National College "Mihai Eminescu"

5 MiHAi EMINESCU STREET

SATU MARE 440014, ROMANIA

Email address: ovidiutiberiu@yahoo.com 